\title{
Teoría de los acontecimientos afectivos: Revisión de su impacto y desarrollos en el estudio del afecto en las organizaciones
}

\author{
Manuel Velasco ${ }^{1, a}$, Jose Navarro ${ }^{a}$, Rita Rueff-Lopes ${ }^{b}$
}

Universitat de Barcelona, Barcelona, Espanhaa, Universitat Ramon Llull, Barcelona, Espanha ${ }^{b}$

\section{Palabras-clave:}

teoría de los acontecimientos

afectivos;

emociones;

estados de ánimo;

acontecimientos laborales

actitudes laborales.

\section{Resumen}

La Psicología del Trabajo y de las Organizaciones presta una atención especial al estudio del afecto. Una de las propuestas más influyentes es la teoría de los acontecimientos afectivos (Weiss \& Cropanzano, 1996). La teoría explica cómo determinados acontecimientos, influenciados por el ambiente externo, pueden provocar respuestas emocionales que determinarían el comportamiento y las actitudes en el lugar de trabajo. En este estudio se revisa la influencia que ha tenido esta teoría. Tras una búsqueda en PsycINFO ${ }^{\circ}$ se encontraron 32 artículos que pusieron a prueba tal teoría. Mediante el análisis de esta producción científica se detectó: (a) el auge que la teoría ha tenido en los últimos años (especialmente desde 2008); (b) el fuerte apoyo a las relaciones que la teoría propone, en especial a la cadena acontecimientos-afecto-actitudes, que constituye el núcleo de la teoría; y (c) la aparición de nuevos elementos (i.e., estrategias de afrontamiento) que enriquecen la propuesta original.

Teoria dos acontecimentos afetivos: Revisão do seu impacto e desenvolvimentos no estudo do afeto nas organizações

\section{Resumo}

Atualmente, a Psicologia do Trabalho e das Organizações dedica uma atenção considerável ao estudo do afeto. Neste sentido, uma das propostas mais influentes é a teoria dos acontecimentos afetivos (Weiss \& Cropanzano, 1996). A teoria explica como determinados acontecimentos, influenciados pelo ambiente externo, podem provocar respostas emocionais que influenciam comportamentos e atitudes no local de trabalho. O objetivo deste estudo é efetuar uma revisão meticulosa acerca da influência desta teoria. Através de uma pesquisa inicial no PsyclNFO ${ }^{\circ}$ foram encontrados 32 artigos que testaram diretamente a teoria. Através da análise desta produção científica se detectou: (a) o auge que a teoria atingiu nos últimos amos (em especial desde 2008); (b) o forte suporte às relações que a teoria propõe, sobretudo à cadeia acontecimentos-afeto-atitudes, a qual constitui precisamente o núcleo da teoria; e (c) a aparição de novos elementos (i.e., estratégias de coping) que vêm enriquecendo a proposta teórica original.

Affective events theory: A review of its influence and developments in the study of affect in organizations

\section{Keywords:}

affective events theory;

emotions:

moods;

work events;

work attitudes.

\begin{abstract}
Industrial and Organizational Psychology is now presenting much more interest in the study of affect at work. One of the most influential proposals in this regard is affective events theory (Weiss \& Cropanzano, 1996). The theory explains how certain events, influenced by the environment, can cause affective responses that, in turn, determine behavior and attitudes in the workplace. We will review here the influence this theory has had. In searching PsycINFO, 32 papers were found that have tested the theory. In the analysis of this scientific production we could infer: (a) the peak that the theory has had in recent years (especially, since 2008); (b) strong support of the relationships proposed by the theory, especially for the events-affect-attitudes chain, which represents precisely the core of the theory; and (c) the emergence of new elements (i.e., emotion-focused coping) that are enriching the original proposal.
\end{abstract}

\footnotetext{
Endereço para correspondência:

Gran Via de les Corts Catalanes, 585, 08007 Barcelona, Espanha.E-mail: manuvesilens@gmail.com j.navarro@ub.edu

Como citar este artigo:

Velasco, M., Navarro, J., \& Rueff-Lopes, R. (2017). Teoría de los acontecimientos afectivos: Revisión de su impacto y desarrollos en el estudio del afecto en las organizaciones. Revista Psicologia: Organizações e Trabalho, 17(1), 30-38. doi: 10.17652/rpot/2017.1.12106
} 
En 1996 Weiss y Cropanzano redactaron lo que a día de hoy muchos autores consideran uno de los textos de mayor influencia para el entendimiento del papel que juegan las experiencias afectivas en las organizaciones (e.g., Ashton-James \& Ashkanasy, 2005). Se trata de la teoría de los acontecimientos afectivos (affective events theory, conocida también como AET) consistente en un profuso marco de trabajo para el estudio del afecto en la vida laboral, una guía para orientar la investigación mediante la integración de estudios y teorías previas al identificar puntos clave y aportar directrices para la comprensión y análisis de los estados afectivos en el trabajo (Weiss \& Beal, 2005).

Uno de los aspectos más destacados de la AET es su apuesta por la necesidad de ahondar en el conocimiento de las estructuras y procesos subyacentes a las experiencias afectivas en contextos laborales, sobre todo enfatizando su naturaleza cambiante y dinámica, a la vez que presta atención a los efectos de estas experiencias afectivas sobre el comportamiento y actitudes del trabajador (Weiss \& Rupp, 2011). Por ello, creemos importante realizar una revisión exhaustiva, sistemática y organizada de aquellas publicaciones que han trabajado utilizando las bases argumentales expuestas en la $A E T$, comprobar y analizar qué soporte empírico ha recibido la teoría y, finalmente, poner de manifiesto cuáles pueden ser las rutas para futuras investigaciones que utilicen la AET. Éstos serán los objetivos de este trabajo.

Para abordarlos, el presente artículo tendrá la siguiente estructura. Primero, detallaremos las principales características de la AET como modelo teórico para el estudio del afecto en contextos laborales. Segundo, describiremos los objetivos específicos perseguidos por el estudio empírico que hemos realizado. Tercero, detallaremos el estudio de revisión llevado a cabo presentando el procedimiento seguido y los resultados encontrados. Seguiremos con una amplia discusión sobre los resultados prestando especial atención al soporte empírico que ha recibido la teoría y aquellos aspectos pendientes de exploración. Finalizaremos con propuestas a considerar por la investigación futura que quiera aplicar la AET. Esperamos con todo ello clarificar y estimular la aplicación de la AET en el estudio del afecto en las organizaciones.

\section{La teoría de los acontecimientos afectivos}

La comprensión del papel del afecto en el comportamiento organizacional es una de las tendencias actuales más remarcables en la psicología del trabajo y de las organizaciones. Basta con prestar atención a índices de últimos números de revistas populares en el área, como la Journal of Applied Psychology o la Journal of Organizational Behavior, o a manuales recientemente publicados (e.g., Kozlowski, 2012) para entender la relevancia e interés del tema. Por poner un ejemplo concreto, el PANAS (Positive and Negative Affect scales, Watson, Clark, \& Tellegen, 1988) es una de las herramientas de evaluación más profusamente utilizadas, recibiendo más de 10.000 citas en la base PsycINFO', y siendo también mayoritario su uso en la psicología aplicada al mundo laboral lo que desvela el interés por el afecto en ese área de la psicología.

La teoría de los acontecimientos afectivos, en adelante (AET), justamente es un modelo teórico que resume los elementos, la estructura y los procesos implicados para entender las experiencias afectivas en contextos laborales. También es una teoría interesada en la comprensión de las influencias de estas experiencias afectivas en el comportamiento y actitudes del trabajador. El modelo teórico, apreciable en la Figura 1, propone una serie de elementos nucleares para entender la dinámica afectiva del trabajador que pasamos a detallar a continuación.

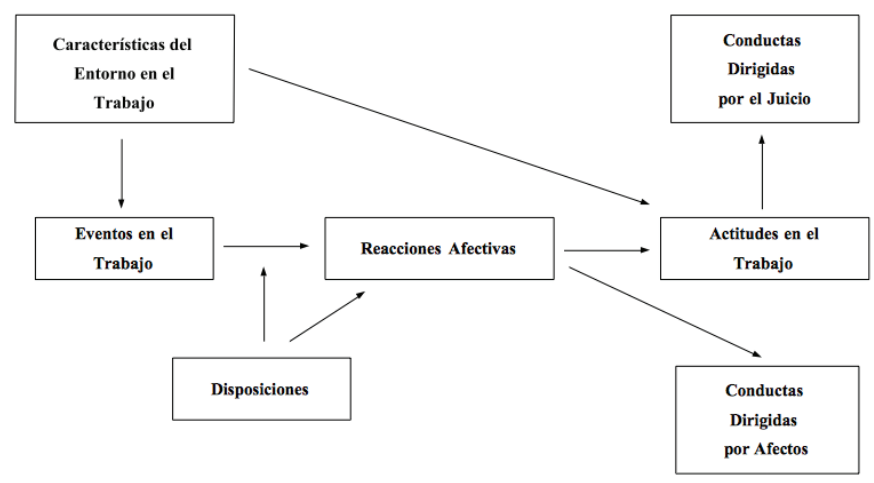

Figura 1. Macroestrucrura de la AET. Fuente: Adaptado de Weiss y Cropanzano (1996).

\section{El afecto en el trabajo: Reacciones afectivas}

En el corazón del modelo propuesto por la AET se ubican todos aquellos elementos y procesos afectivos que surgen a consecuencia de algún acontecimiento específico dentro del contexto laboral. No se trata entonces de cualquier tipo de afecto sino, en concreto, de aquellos estados más o menos intensos, más o menos duraderos y más o menos placenteros que se originan como reacción a uno o más acontecimientos ocurridos en contextos laborales. La AET organiza conceptualmente estas reacciones distinguiendo dos formas esenciales: las emociones y los estados de ánimo (EA). Aun que con distinto nivel de detalle y estabilidad temporal, ambas comparten cualidades suficientes como para inscribirse dentro del mismo espacio dentro del modelo. Sin embargo, sus características diferenciadoras se deben tener en cuenta a la hora de ser estudiadas.

Basándose en los trabajos de Frijda (1993) y Morris (1989), la AET destaca la comparación más común entre EA y emociones que suele hacerse en base a tres características: intensidad, duración y especificidad en relación a un objeto. En este sentido los EA, si se comparan con las emociones, suelen ser más duraderos, menos intensos y más bien difusos respecto a un objeto en particular. No obstante, en estricto rigor, las dos primeras características son poco generalizables y, a menudo, pueden ser de cuestionada utilidad ya que se ha visto que los EA, en algunos casos, varían considerablemente en cuanto a su duración así como en intensidad y aún así tener los efectos atribuidos a la experiencia real de un EA. Esta última observación, nos deja sólo una característica que realmente permitiría diferenciar EA de emociones: la especificidad.

Para la AET tanto las emociones como los EA surgen como una respuesta ante un acontecimiento, ahora bien, en general, los EA no son siempre una respuesta específica a un acontecimiento concreto, por ello sus causas son más difusas que en el caso de las emociones discretas (e.g., Barsade \& Gibson, 2007). Por último, para la AET el afecto no puede entenderse como un rasgo estable siendo por ello lo fundamental la experiencia del afecto como algo dinámico que va variando a lo largo del día a día en el trabajador.

\section{Acontecimientos con significación afectiva}

$Y$, ¿qué desencadena estos EA o emociones en el trabajador? Según la AET dentro del contexto organizacional se están produciendo continuamente eventos externos al individuo, de entre los cuáles los hay que tienen algún grado de significación afectiva. Los autores enfatizan la idea de que dichos acontecimientos significativos son causas proximales de las reacciones emocionales que se dan en el trabajo a la vez que serían causas distales de comportamientos y actitudes laborales. De este modo, las reacciones afectivas 
serían mediadores (mecanismo por el cuál un proceso influye en un segundo proceso) entre los acontecimientos y el comportamiento.

La fundamentación que utiliza la AET para explicar el proceso por el cual ciertos eventos pueden llegar a generar cambios en el afecto se fundamenta en la amplia investigación cognitiva que ha encontrado como la cognición es clave para entender las reacciones afectivas (e.g., Lazarus, 1991; Ortony, Clore, \& Collins, 1988; Roseman, 1984; Smith \& Ellsworth, 1987; Stein, Tribasso, \& Liwang, 1993 citados por Weiss \& Cropanzano, 1996) y que coincide en proponer que existe un proceso compuesto por dos fases de evaluación del evento una vez que éste se ha producido: primero, el evento es evaluado en función de su relevancia para el bienestar del trabajador (evaluación realizada en términos genéricos de positivo-negativo) o por su estrecha relación con las metas y los valores personales (Frijda, 1993) - estos eventos representarían auténticos acontecimientos ${ }^{2}$ para la vida del trabajador; y segundo, una evaluación más específica o secundaria a modo de análisis interpretativo del sentido en relación al evento, elicitante y centrada en las consecuencias y atribuciones del mismo. Es en esta segunda fase cuando claves específicas del entorno y de la persona son consideradas dando lugar a respuestas emocionales discretas como la tristeza, la rabia o la alegría (Ortony et al., 1988; Smith \& Pope, 1992).

\section{Actitudes laborales}

Siguiendo la teoría de las actitudes (Eagly \& Chaiken, 1993), la AET contempla las actitudes como evaluaciones generales en términos de favorabilidad-desfavorabilidad que están influenciadas tanto por las experiencias afectivas con el objeto actitudinal como por las características del propio entorno de trabajo (e.g., el clima o cultura organizacional). La teoría actitudinal ha propuesto cogniciones y afectos como los componentes más clásicos de las actitudes. Ahora bien, la AET nos enfatiza que a la hora de hablar de actitudes laborales han predominado el enfoque cognitivo. El ejemplo paradigmático serían los estudios sobre satisfacción laboral que mayoritariamente han reducido, según Weiss y Cropanzano (1996), esta actitud a sus componentes cognitivos. Por ello, la AET ha enfatizado también el rol que el afecto (i.e., emociones y estados de ánimo) tiene sobre estas actitudes.

Según la AET, el trabajador, a menudo, construye sus actitudes al momento en que éstas son requeridas consultando en su memoria de experiencias anteriores la información relevante, presumiblemente experiencias afectivas con el objeto actitudinal, así como información basada en sus cogniciones (e.g., creencias; Wilson \& Hodges, 1992). Nunca se usa toda la información retenida, sino que la persona genera sus actitudes a partir de un subconjunto de dicha información que, a su vez, también es influenciada por factores contextuales. En este sentido, debido a que los procesos cognitivos son una parte importante de las reacciones afectivas es mejor hacer la distinción entre las influencias que representan experiencias emocionales con el objeto actitudinal de aquellas influencias que se relacionan con el conjunto de creencias acerca de las características del objeto (Breckler \& Wiggins, 1989). Esto explicaría el que las actitudes pueden ser bastante inestables ya que los factores situacionales también van a determinar parte de la información

\footnotetext{
Según la RAE (Real Academia Española) un evento es una 'eventualidad, hecho imprevisto, o que puede acaecer' o bien un 'suceso importante y programado, de índole social, académica, artística o deportiva'. Por su lado, un acontecimiento se referiría a 'hecho o suceso, especialmente cuando reviste cierta importancia'. Es esta acepción de la importancia, y de la no necesaria programación, lo que ha hecho decantarnos por la traducción de la AET al español como teoría de los acontecimientos afectivos.
}

que será utilizada. La inestabilidad, o variabilidad, es una clave en la AET: las experiencias afectivas son fluctuantes en el tiempo, los acontecimientos cambian y el posterior comportamiento y actitudes del trabajador también lo harán.

\section{Comportamientos dirigidos por el afecto vs comportamientos dirigidos por las actitudes}

La teoría sugiere que para comprender la relación entre el afecto y el comportamiento del trabajador habría que incluir la distinción entre comportamientos dirigidos por el afecto (CDA) y aquellos comportamientos dirigidos por el juicio o las actitudes (CDJ). Los primeros son comportamientos que se dan en el trabajo como respuesta directa a las experiencias afectivas siendo en estos casos cuando el patrón particular de respuestas afectivas puede tener importantes efectos, por ejemplo en el desempeño laboral, sin que exista mediación en la relación entre las experiencias afectivas y las actitudes. La AET remarca que los niveles de afectividad pueden fluctuar por lo que se podría esperar que estos CDA sean de corta duración y de gran variabilidad, razón por la que cualquier relación será mejor captada mediante análisis que evalúen la congruencia de los patrones fluctuantes del afecto y del desempeño (e.g., empleando estudios intra-participante). Los CDA estarían influidos por estados de ánimo y emociones discretas, que han sido activadas por el contexto laboral, y serían unos comportamientos distintos de las actitudes que un trabajador haya podido generarse sobre su trabajo $u$ organización.

Por otro lado, la AET reconoce otro tipo de comportamientos, los comportamientos dirigidos por las actitudes o el juicio (CDJ), que estarían directamente influenciados por la evaluación general del propio trabajo u organización y, por ello, cualquier relación entre los niveles de afecto y estas conductas sí será mediada por las actitudes que se tengan en relación al trabajo. Suelen ser comportamientos que derivan de decisiones bien consideradas, específicamente aquellas donde esa evaluación general del trabajo u organización entra en dicha decisión. Los CDJ serían, por tanto, comportamientos más estables que los anteriores CDA.

\section{Disposiciones}

Para cuando se redactaba la AET gran parte de las investigaciones sobre personalidad y satisfacción laboral (SL) estaban centradas en los rasgos de afectividad positiva (AP) y afectividad negativa (AN) ya que ambos permiten hacer predicciones de tendencias emocionales generales de las personas. Varios estudios (Cropanzano, James, \& Konovsky, 1993; Levin \& Stokes, 1989; Watos \& Slack, 1993; citados por Weiss \& Cropanzano, 1996) mostraron que ambos rasgos (AP y AN) correlacionaban con las actitudes hacia el trabajo (SL). La AET, sin desmerecer estos esfuerzos, amplía esta idea agregando que realmente el papel que juegan las disposiciones es el de influenciar la forma en que los acontecimientos producen reacciones afectivas; es decir, que dichas disposiciones serían moderadores en la relación entre acontecimientos y afecto. En este punto los rasgos afectivos o de personalidad figuran como predisposiciones latentes que ayudan a organizar el escenario a los individuos. Estos rasgos son, por tanto, sólo disposiciones afectivas y no la experiencia en sí misma, lo cual quiere decir que predisponen al individuo a responder con mayor o con menor intensidad a los eventos con significación afectiva.

\section{Características del entorno laboral}

En la teoría se da soporte al planteamiento sobre los individuos como agentes activos que interactúan de forma dinámica con su 
entorno influenciando y dejándose influenciar por éste (Kohn \& Schooler, 1982). Para la AET, el ambiente laboral puede tener un efecto indirecto sobre las experiencias afectivas, aumentando o bien disminuyendo las probabilidades de que determinados acontecimientos ocurran y consiguientemente que generen una reacción emocional. A la vez, las características del entorno también podrían ejercer un efecto directo sobre los componentes cognitivos de las actitudes. Sin embargo, ha de tenerse en cuenta que esta doble influencia está expresada en términos generales por lo que no hay que confundir esta idea con la de que cada característica del ambiente en el trabajo incluye siempre influencias directas e indirectas a la vez.

\section{La estructura episódica de las experiencias afectivas}

La AET pone énfasis en el interés por el estudio de las estructuras episódicas de las experiencias afectivas en el trabajo, en sus comienzos y finales. Todo ello sobre la base de que la vida, y no sólo la vida emocional o laboral, está estructurada episódicamente (Weiss \& Beal, 2005). En este sentido, la clave del concepto de episodio emocional es que éste representa el flujo y reflujo de la experiencia afectiva a través del tiempo. Así, mientras cada uno de los acontecimientos durante el episodio puede ser descrito en términos discretos, éste en sí mismo posee una coherencia y un conjunto de características que sugieren que debe ser tratado como una unidad de análisis.

Los episodios de la experiencia emocional exhiben una estructura en la que se que incluyen momentos pico, rastros persistentes y recurrencias, por lo que representarlos mediante una simple agregación de experiencias en el tiempo dista de ser apropiado (Beal \& Weiss, 2003). El afecto para la AET, tanto como variable dependiente así como independiente, requeriría siempre de la consideración de sus fluctuaciones a través del tiempo (Weiss \& Rupp, 2011).

\section{Objetivos de la investigación}

Habiendo descrito los elementos más relevantes de la $\mathrm{AET}$, los objetivos que organizan y dirigen el presente trabajo son los siguientes: primero, realizar una revisión exhaustiva y sistemática de los artículos científicos que hayan utilizado la AET como base y guía para su desarrollo; segundo, revisar aquellos trabajos que ponen a prueba los componentes y relaciones del modelo de la AET para extraer conclusiones acerca de la validez del modelo propuesto; $y$ tercero, presentar nuevas propuestas de investigación en consonancia con las directrices teóricas y metodológicas que definen la AET así como considerando la investigación ya previamente desarrollada.

\section{Método}

\section{Muestra y procedimiento}

La muestra de partida estuvo compuesta por todos los artículos que aparecen en la base de datos PsycINFO ${ }^{\circ}$ y que han citado la publicación original de la AET de Weiss y Cropanzano (1996). Decidimos basarnos en PsycINFO ${ }^{\circ}$ al ser ésta la principal base de datos internacional de referencia en el área de la psicología. Como es sabido PsycINFO incluye cerca de 4 millones de publicaciones en ciencias del comportamiento y sociales, áreas de conocimientos donde claramente se incluye la temática del afecto al estar éste referido a una de las manifestaciones más claras del comportamiento humano. Una vez dentro de PsycINFO', y localizada la publicación original, bastó con desplegar en el motor de búsqueda la opción cited by para conocer todos aquellos artículos que hayan referenciado la AET. Después, nos quedamos con las publicaciones que hubieran aparecido en revistas con procesos de revisión por pares como filtro para trabajar con documentos que han sido evaluados externamente. La búsqueda se realizó en los meses de octubre y noviembre de 2014 y se obtuvieron un total 187 artículos.

Tras esta búsqueda general decidimos incluir de forma complementaria algunos trabajos referenciados por Weiss y Beal (2005), en su actualización de la AET, así como algunos otros trabajos que fueron encontrados en el transcurso de la revisión de los artículos, específicamente aquellos de corte empírico que ponían a prueba elementos o procesos del modelo, pero que no figuraban en los resultados arrojados por el motor de búsqueda de PsycINFO ${ }^{\circ}$. Esta parte de la muestra constituye el $15,6 \%$ del total final de documentos considerados.

Para facilitar el análisis se optó por organizar la información de cada artículo en fichas de contenido. Seguidamente, utilizamos un segundo criterio de inclusión dejando fuera aquellos trabajos que sólo citaban la AET como soporte específico de algún argumento teórico pero sin que dichos trabajos se hayan centrado o basado en ella, de forma que sólo quedaron aquellas publicaciones que explícitamente hayan utilizado la AET como marco de trabajo principal. De todo este proceso, y como muestra final de trabajo, nos quedamos con 32 artículos, 23 de ellos artículos empíricos, 2 meta-análisis y 9 artículos de revisión o reflexión teórica.

\section{Análisis}

Con la información ya organizada se realizó un análisis de contenido para todos los artículos sobre los siguientes aspectos: autor, año de publicación, revista o fuente, país y variables estudiadas. Además, de forma específica para aquellos artículos de corte empírico, se analizó el tipo de diseño empleado, las herramientas de medida, los elementos y relaciones incluidas en alguna de las hipótesis o preguntas de investigación así como las propuestas nuevas o alternativas respecto al modelo original. En consonancia con las intenciones manifestadas en nuestros objetivos, hemos decidido enfatizar el análisis en aquellos artículos que o bien pongan a prueba alguna parte o relación del modelo de la AET o bien que, utilizando éste como guía o base, propongan nuevas relaciones o elementos.

\section{Resultados}

Como anticipamos, el total de artículos encontrados en la base de datos PsycINFO ${ }^{\circ}$ que citan la AET fue de 187. Atendiendo a la evolución temporal del número de artículos que la han citado pueden observarse dos claros periodos: hasta el 2007, en el que la AET recibe pocas citas, unas cinco de promedio por año; $y$ desde el 2008 hasta la actualidad, donde las citas se incrementan considerablemente pasando en los últimos años a recibir 25-30 citas anuales. De estos 187 artículos totales que citan la fuente original de la AET 32 representan puestas a prueba o desarrollos sobre la propia teoría. Nos centraremos en éstos de aquí en adelante.

Al revisar la autoría de las publicaciones los resultados permiten constatar que, considerando sólo aquellos autores que han publicado más de un trabajo dentro de los 32 artículos, no existen grandes diferencias ya que, salvo Neal M. Ashkanasy con tres artículos publicados, nadie más supera las dos publicaciones. En este sentido lo autores con más de una publicación son Dan Ispas, Edward L. Levine, Cynthia D. Fisher, James M. LeBreton, John F. Binning, Christian Scheltt, René Ziegler, Arthur P. Brief y Charmine E. J. Härtel. Se observa además que predominan las publicaciones empíricas que representan el $80 \%$ dentro de los autores mencionados. 
Todos exhiben únicamente publicaciones de corte empírico salvo los casos de Arthur P. Brief quién aporta revisiones teóricas y Neal M. Ashkanasy con dos empíricas y una teórica.

En cuanto a las fuentes de publicación se puede constatar que, de las 22 que componen el total, una serie de ellas cuenta con dos o más publicaciones. Son las siguientes: Human Performance, Australian Journal of Management, Journal of Applied Psychology, Journal of Vocational Behavior, Human Relations, Organizational Behavior and Human Decision Processes, Motivation and Emotion y Journal of Personnel Psychology. Se trata de revistas, como vemos, de alta reputación en el área que pertenecen a los cuartiles 1 o 2, dentro del Journal Citation Report de la Social Sicences Citation Index. En promedio, el factor de impacto de éstas revistas es 2,08 (rango entre 0,80 y 4,78 ) lo que permite hacernos una idea de la importancia del tema dentro de la literatura organizacional.

En relación a la participación por países destaca Estado Unidos ( $n=18)$ como el país donde mayor cantidad de autores han realizado publicaciones, siendo el doble que en el caso de Australia $(n=9)$ que le sigue en segundo lugar. Ambos llevan una considerable ventaja respecto al resto de los países los que, salvo el caso de Alemania $(n=4)$ e Inglaterra $(n=2)$, sólo cuentan con un autor con una publicación. Por otro lado, el análisis de las variables estudiadas revela que existe un bajo grado de consenso sobre las etiquetas utilizadas para referirse, en muchas ocasiones, a los mismos procesos psicológicos. En concreto, hemos contabilizado un total de 83 variables. De aquellas que han aparecido con mayor frecuencia, la variable más estudiada ha sido la satisfacción laboral $(n=13)$, seguida de la afectividad negativa $(n=10)$ y afectividad positiva $(n=9)$. Creemos que es destacable también el que la mayoría de las variables más estudiadas coinciden con componentes afectivos tanto de rasgo (e.g., afectividad positiva o negativa) como de estado (e.g., EA o emociones).

\section{Artículos empíricos: Diseños, herramientas de evaluación y relaciones estudiadas}

Nos centraremos ahora en aquellos trabajos empíricos $(n=23)$ que nos aportan información relevante sobre la validez de la teoría y de las relaciones que ésta propone. La mayoría de estos estudios han utilizado diseños de tipo transversal, principalmente con medidas entre-sujetos; sin embargo, se debe destacar que un 39,1\% sí han incluido medidas intra-sujeto, diseño que, sin lugar a dudas, es más coherente con los postulados de la AET.

Nos encontramos con una amplia variedad de herramientas aplicadas para la medición de las variables y constructos. Sin embargo, al contabilizar su frecuencia de uso hemos constatado que la gran mayoría sólo fue utilizada en no más de un estudio. No obstante, puede destacarse el uso de algunas herramientas que han sido utilizadas al menos en tres estudios diferentes. En concreto, PANAS (Watson et al., 1988), utilizado comúnmente para medir la afectividad positiva y negativa en el trabajo, y que fue aplicado en nueve estudios dentro de los que se incluyen también algunos casos en los que se aplicó la versión extendida PANAS- $X$ (Watson \& Clark, 1994). Segundo, para la medición de emociones específicas en el ámbito laboral se aplicó la Job Emotion Scale (JES; Fisher, 1997) en cuatro estudios. Por último, y con tres aplicaciones contabilizadas, figura la Job Satisfaction Subscale of the Michigan
Organizational Assessment Questionnaire (MOAQ-JSS; Cammann, Fichman, Jenkins, \& Klesh, 1983) para medir la satisfacción laboral.

Desde el discurso de la AET se entiende que, más que las partes aisladas del modelo, lo que adquiere mayor relevancia son las relaciones y procesos subyacentes. En este sentido, en la Tabla 1 mostramos las relaciones estudiadas así como el respaldo empírico que han recibido. Es posible apreciar que la mayoría de los esfuerzos se concentran en torno a la relación entre las reacciones afectivas y las actitudes (19,5\%). Estudios como el llies y Judge (2002) que miden la relación entre el EA, tanto positivo como negativo, con actitudes como la satisfacción laboral obteniendo resultados significativos en casi la totalidad de los estudios aportando importante evidencia favorable a esta relación. Si bien este tipo de estudios son los más representativos aunque también son interesantes aquellos que para estudiar esta relación dan un paso más en complejidad, expandiendo la simple dicotomía positivo-negativo y desglosan el afecto en emociones discretas como en Levine et al. (2011) quienes en un estudio transnacional observaron diferencias en los resultados en función de las emociones específicas (i.e., alegría, orgullo, ansiedad, envidia) y encontraron que éstas eran mejores predictores de la satisfacción laboral que el afecto contemplado de forma general.

La relación entre acontecimientos y reacciones afectivas también ha destacado dentro de la literatura $(17,1 \%)$. Quizás uno de los ejemplos más interesantes se encuentra en Zohar, Tzischinski y Epstein (2003) quienes se han centrado en los acontecimientos que ocurren día a día en la vida del trabajador encontrando que éstos pueden actuar como causas o estímulos proximales para la evocación de reacciones emocionales discretas. Específicamente, demostraron que eventos disruptivos se relacionan positivamente con afectos negativos y lo mismo ocurre entre eventos estimulantes y afectos positivos. Otra relación con destacada presencia en los estudios es la que hay entre las reacciones afectivas y las CDA $(17,1 \%)$. En el ya mencionado estudio transnacional de Levine et al. (2011) no sólo demostraron esta relación sino que además pudieron comprobar que variaba en función del tipo de emoción así como del tipo de conducta que se estuviese midiendo. Así, obtuvieron resultados claramente diferenciados cuando se medían las relaciones de las diferentes emociones discretas contempladas en su estudio con variables como los comportamientos de ciudadanía organizacional o los comportamientos contraproducentes en el trabajo.

La relación entre las disposiciones y las reacciones afectivas también ha destacado en presencia en esta muestra de artículos $(12,9 \%)$ y profundizar sobre la misma era uno de los objetivos del trabajo realizado por Grandey, Tam y Brauburger (2002). Estos autores investigaron el tipo de vínculo que hay entre las disposiciones afectivas AP y AN y las reacciones emocionales específicas del mismo tono hedónico que se dan en el trabajo obteniendo resultados que demostraban que la AN era un buen predictor de emociones negativas generales que se daban en el entorno del trabajo; no obstante sólo obtuvieron soporte parcial al poner a prueba la relación entre la AP y las reacciones afectivas positivas.

La relación entre el entorno laboral y los acontecimientos es la única que no ha sido puesta a prueba en ningún estudio. También destacaríamos la relación entre entorno laboral y las actitudes en el trabajo $(2,4 \%)$ que como ya se ha mencionado antes, sólo fue revisada en el trabajo de Fisher (2002). 
Tabla 1

Relaciones puestas a prueba

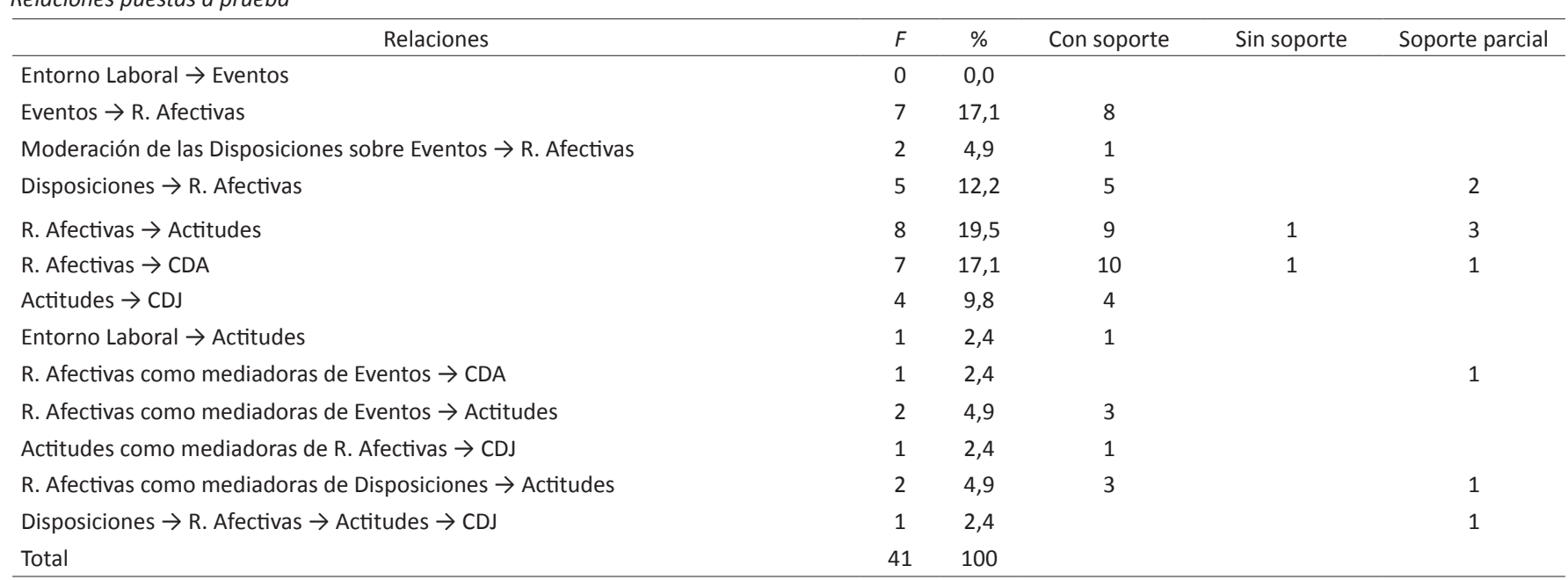

Si bien gran parte de los estudios someten a prueba más de una relación propuesta por la AET, en general esto se ha hecho de forma parcelada sin ir más allá de la relación entre dos elementos del modelo que hemos ido apuntando en los párrafos anteriores. Frente a ello, adquieren particular interés las pocas excepciones, y que sólo aportan el $17 \%$ de entre las totales, que han sometido a prueba segmentos más extensos del modelo. Por ejemplo, la mediación de las reacciones afectivas en la relación entre los acontecimientos y las actitudes. Un caso revisado se encuentra en el trabajo realizado por Miralles, Navarro y Unger (2015) donde se demostró el rol mediador del afecto, tanto positivo como negativo, entre la evaluación de los acontecimientos en el trabajo y la dedicación al trabajo. Finalmente, en los trabajos revisados la gran mayoría de ensayos realizados para poner a prueba las relaciones del modelo han obtenido resultados positivos salvo en unos pocos casos donde alguna hipótesis no obtuvo soporte o bien sólo soporte parcial (ver Tabla 1).

\section{Propuesta de relaciones alternativas}

El $52,1 \%$ de los estudios empíricos revisados integran propuestas alternativas respecto a las originales del modelo. Hemos recogido todas estas nuevas propuestas en la Figura 2. Se trata de relaciones que siguen las directrices teóricas de la AET, pero que no figuraban en el modelo original. En esta línea, destaca el estudio de la relación entre las disposiciones y las actitudes ya que se puso a prueba en tres estudios. Sobre todo hemos observado relaciones que se suman sin disrupción a las originales, como en Iverson y Deery (2001) quienes además de demostrar que la SL es predictor de ciertas conductas de absentismo, encontraron que las disposiciones afectivas de los individuos también se vinculaban a dichas conductas e influían en la frecuencia de aparición de las mismas. En este caso, las categorías de componentes no difieren con las de la AET ya que se trata de AP y AN (disposiciones) y las conductas de absentismo (CDJ) por lo que la novedad se encuentra específicamente en la relación que se propone entre ambas ya que no se encuentra contemplada en el modelo original.

Otro ejemplo se encuentra en Lebreton, Binning, Adorno y Melcher (2004) quienes estudiaron las diferencias entre la estabilidad emocional y tres de los cinco grandes (extraversión, responsabilidad y afabilidad) en cuanto su capacidad para predecir ciertas actitudes negativas que se dan en el trabajo, encontrando que la estabilidad actúa como mejor predictor de determinadas actitudes laborales negativas. Otra interesante propuesta complementaria se aprecia en Gooty, Gavin, Ashkanasy y Thomas (2014) quienes adhieren una nueva variable (i.e., las estrategias de afrontamiento centradas en la emoción o EFC) sobre el que se articulan nuevas relaciones sin alejarse de los fundamentos de la AET sino, por el contrario, utilizándolo como marco de trabajo. Hemos incluido en la Figura 2 este nuevo componente que en realidad es un concepto ampliamente abordado en psicología y, en especial, en el estudio del estrés que conviene recordar es considerada también una respuesta afectiva.

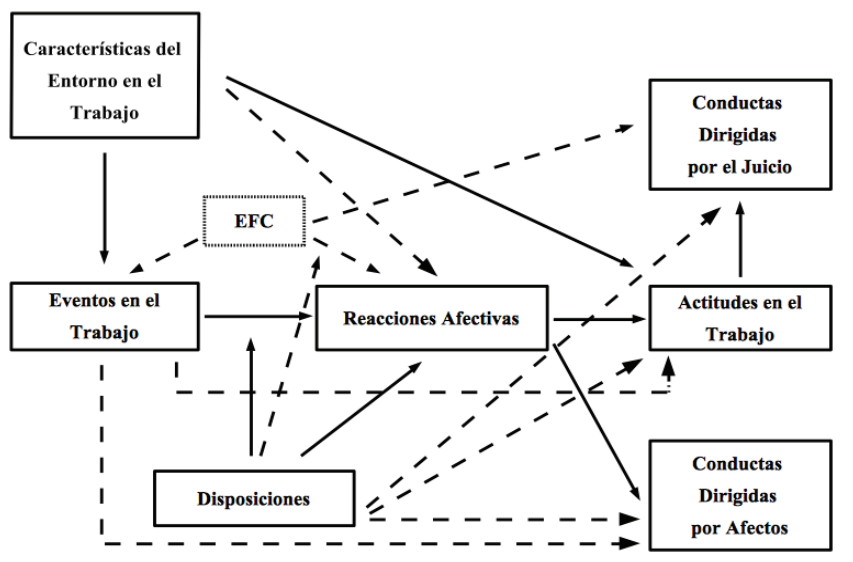

Figura 2. Nuevas propuestas sobre el modelo original de la AET. Nota: las flechas y cajas con línea continua representan al modelo original de la AET; las flechas y cajas discontinuas recogen nuevas variables y relaciones sobre le modelo original.

\section{Discusión}

En general, los resultados encontrados están en sintonía con lo que cabría esperar desde la mirada amplia de la AET. Salvo en unas pocas y aisladas excepciones, los objetivos de las investigaciones que han propuesto alguna contrastación de modelo obtuvieron resultados en la dirección esperada y sin alejarse de los aspectos que exhiben más valor implícito en los argumentos y en el discurso general de la AET. Por ejemplo, la cadena principal expuesta por la AET (acontecimientos que desencadenan afectos y, éstos a su vez, desencadenan actitudes) cuenta con un claro apoyo empírico (17 hipótesis que se confirmaron frente a cuatro hipótesis sin soporte o con soporte parcial).

Por otro lado, la contribución de la AET para entender comportamientos dirigidos por la actitud (como sería el caso de la satisfacción laboral) también se ha mostrado con un importante soporte 
en las investigaciones (10 hipótesis que se confirmaron frente a dos hipótesis sin apoyo o con apoyo parcial). Conviene recordar ahora que entre las motivaciones originarias para la propuesta de la AET estuvo justamente la de presentar una comprensión más amplia de la satisfacción laboral como una de las manifestaciones actitudinales de mayor tradición en la psicología organizacional.

Frente al soporte recibido también conviene señalar como algunas de las relaciones propuestas no se han puesto a prueba. Este último es el caso de la relación entre entorno laboral y acontecimientos que no se ha estudiado aún. Hay aquí, por tanto, una demanda para la investigación futura pues, como propone la AET, parece sensato pensar que determinados ambientes laborales estimularán la aparición de ciertos eventos afectivos. Por ejemplo, en el denominado trabajo emocional parece evidente que influirán políticas y prácticas organizacionales.

\section{Evoluciones sobre el modelo original}

Ya se ha dicho que la AET más que una teoría o un modelo para poner a prueba es una guía que pretende orientar el estudio del afecto en las organizaciones (Weiss \& Beal, 2005) por lo que no ha de extrañarse que en esta revisión de artículos surjan nuevas propuestas que abarcan nuevos elementos y relaciones. Tal y como ya quedó expuesto en los resultados, una parte importante de los trabajos empíricos no se ha ceñido únicamente a la postura del modelo original sino que además han integrado las aportaciones conceptuales de la AET con otras teorías relacionadas generando así propuestas alternativas que complementan el modelo (ver Figura 2).

Con la virtud de no alterar en profundidad la estructura original, pero a la vez sumando nuevas relaciones entre los elementos, lo más representativo han sido investigaciones con aportaciones complementarias de esta índole. No obstante, una de estas propuestas alternativas que va un paso más allá, exhibiéndose como una de las más interesantes, se encuentra en el trabajo anteriormente mencionado de Gooty et al. (2014). Tal y como se aprecia en la Figura 2 los autores han aportado, además de nuevas relaciones, la intervención del EFC como un elemento más dentro del modelo. De este modo añaden más contenido, específicamente sobre la fase general de evaluación que ocurre entre cómo se vive el evento y su reacción posterior. Estos autores estudiaron con éxito el efecto de moderación de la inteligencia emocional conceptualizada como una habilidad (ability-based emotional intelligence; $\mathrm{ABEl}$; Mayer et al., 1999 citado por Gooty et al., 2014), sobre la relación entre el EFC y ciertas emociones específicas en el trabajo, y lo hicieron utilizando gran parte del contenido teórico que se expone en la AET con especial interés en aportaciones que fundamentan el proceso de evaluación de los eventos. En definitiva, el resultado apreciable ha sido el de un poderoso nuevo ingrediente para complementar el modelo original y con ello se abre una amplia ventana a futuras investigaciones para la inclusión de otros nuevos elementos o componentes.

Nuevos caminos surgen como resultado de ir un poco más adentro hacia la complejidad que define al afecto en el trabajo, dejando así en evidencia la flexibilidad del modelo, lo que a su vez sugiere nuevas rutas de investigación que, sin desvincularse de la propuesta original, pueden aportar un valor añadido a la propia teoría. Sin embargo, esto también pone de manifiesto la necesidad de concretar con mayor solidez un modelo que, según como se mire, puede resultar extremadamente básico y ambiguo, y que desde hace casi 20 años pareciera que no ha seguido madurando. Sea cual fuera el ojo crítico con que se mire, a fin de cuentas, hay que volver a recordar que este modelo es sólo una guía, sin otra ambición más que orientar el estudio del afecto en las organizaciones.

\section{El afecto en el trabajo: ¿'Rasgo o estado?}

Un punto clave que ha de tenerse en consideración a la hora de revisar los trabajos empíricos que han utilizado la AET es la distinción entre rasgos afectivos y estados afectivos. Los estados afectivos, el núcleo de la $\mathrm{AET}$, se caracterizan por la propiedad de fluctuar a través del tiempo y, por ello, para estudiar íntegramente un estado afectivo es necesario considerar esta cualidad definida por el cambio. Por otro lado, cuando se hace referencia a un rasgo entonces estaremos frente a una categoría para el afecto encuadrable dentro de las disposiciones del individuo las que en este caso son más estables y se encuentran estrechamente vinculadas a la personalidad. Sin embargo, la simpleza de la separación conceptual entre el afecto como estado y el afecto como rasgo se contrapone a la complejidad metodológica de llevar esta distinción al plano empírico al punto que ha sido desconsiderada en una parte importante de los estudios revisados.

En este sentido, desde la línea argumental de la AET esperábamos encontrar una amplia mayoría de trabajos con diseños en los que se considerasen las fluctuaciones de las reacciones afectivas que incluyeran diseños de tipo longitudinal acentuando así la importancia de todos aquellos datos que se expliquen por la varianza intra-sujeto. La realidad con la cual que nos hemos encontrado es que sólo un $39,1 \%$ de los trabajos revisados han incluido y considerado estas cuestiones. Esto dejaría una duda razonable no en la teoría en sí, sino en la forma en cómo esta se ha puesto a prueba. No basta con etiquetar una variable de emoción discreta, estado de ánimo, afecto específico del trabajo o de cualquier otra forma que tenga pretensiones de ubicarse dentro de la categoría de reacción afectiva para luego aplicar transversalmente herramientas como el PANAS o el JES a los sujetos investigados y sacar conclusiones amparadas en la AET, ya que sus autores han sido muy claros en subrayar la necesidad de distinguir correctamente cuándo se trabaja con variables estables de cuándo se hace con variables que fluctúan en el tiempo (Brief \& Weiss, 2002).

La AET es un marco teórico que se ajusta bien a las demandas actuales (e.g., Navarro, Roe, \& Artiles, 2015; Roe, 2008) de hacer una investigación que tenga en cuenta la naturaleza temporal y dinámica de los fenómenos abordados por la psicología del trabajo y de las organizaciones. En una reciente revisión sobre el descuido general que ha habido en la investigación acerca del tiempo, Navarro et al. (2015) exponen como gran parte de los conceptos de interés en el área (e.g., motivación, rendimiento, emergencia del liderazgo, procesos de equipos, cultura de empresa, etc.) son procesos caracterizados por su dinámica, con un inicio, un desarrollo y un final. Estos procesos se caracterizan por ser dinámicos, cambiantes, y los diseños de investigación apropiados para su estudio no serían aquellos basados en el estudio de diferencias entre-participantes, hábiles para captar diferencias estables entre individuos (e.g., características socio-demográficas como el género); por el contrario, se precisaría de diseños intra-participante que permiten el seguimiento repetido de las mismas personas a lo largo del tiempo (e.g., muestreos de experiencias) para dar cuenta de los patrones dinámicos que haya en dichos cambios. El afecto, como núcleo de la $A E T$, sería uno de esos procesos cambiantes y dinámicos interesante de estudiar desde el empleo de diseños intra-participante.

Al repasar los puntos clave de la AET el investigador gana conciencia sobre la complejidad que engloba el fenómeno del afecto en el trabajo. La propuesta de Weiss y Cropanzano va dirigida a 
revisar los elementos y procesos de mayor peso, para lo cual el modelo presentado facilita claramente la comprensión del fenómeno afectivo en entornos laborales. La profundidad y amplitud de los contenidos que se abordan en el redactado del texto original hacen de éste un texto con cualidades para guiar y esclarecer conceptos, más que una teoría o modelo específico para poner a prueba y donde constantemente se hace un llamado a reorientar varias de las perspectivas teóricas así como de ciertas tendencias metodológicas que hasta ese entonces han predominado en la literatura.

En general, los trabajos revisados aportan resultados en consonancia con lo esperado, aunque como ya hemos comentado en la discusión aún hay varios ajustes que deben considerarse a la hora de llevar una propuesta como la AET al terreno empírico. Esto, sin embargo, no ha impedido que el interés por esta teoría haya visto un considerable aumento reflejado en la cantidad de artículos que la citan, por ello esperamos que dicho aumento se pueda ver reflejado en un mayor número de trabajos empíricos no sólo para ponerla a prueba sino que además puedan ir afinando el modelo propuesto y lograr así un mayor consenso teórico y metodológico.

\section{Referencias}

*Ashkanasy, N. M., Ayoko, O. B., \& Jehn K. A. (2014). Understanding the physical environment of work and employee behavior: An affective events perspective. Journal of Organizational Behavior, 35(8), 1169-1184. doi: 10.1002/ job.1973

*Ashkanasy, N. M., \& Humphrey, R. H. (2011). Current emotion research in organizational behavior. Emotion Review, 3(2), 214-224. doi: $10.1177 / 1754073910391684$

*Ashkanasy, N. M., Härtel, C. E. J., \& Daus, C. S. (2002). Diversity and emotion: The new frontiers in organizational behavior research. Journal of Management, 28, 307-338. doi: http://dx.doi.org/10.1016/S0149-2063(02)00130-7

Ashton-James, C. E., \& Ashkanasy, N. M. (2005). What lies beneath? A deconstructive analysis of affective events theory. In N. M. Ashkanasy, W. J. Zerbe \& C. E. J. Härtel (Eds.), Research on emotion in organizations (Vol. 1, pp. 23-50). Oxford: Elsevier/JAI Press. doi: 10.1016/S1746-9791(05)01102-8

*Ayoko, O. B., Callan, V. J., \& Härtel, C. E. J. (2003). Workplace conflict, bullying and counterproductive behaviors. The International Journal of Organizational Analysis, 11(4), 283-301. doi: 10.1108/eb028976

Barsade, S. G., \& Gibson, D. E. (2007). Why does affect matter in organizations? Academy of Management Perspectives, 21(1), 36-59. doi: 10.5465/ AMP. 2007.24286163

*Basch, J., \& Fisher, C. (1998). Affective events - Emotions matrix: A classification of work events and associated emotions. School of Business Discussion Papers, Paper 65.

Beal, D. J., \& Weiss, H. M. (2003). Methods of ecological momentary assessment in organizational research. Organizational Research Methods, 6(4), 440-464. doi: $10.1177 / 1094428103257361$

*Becker, T., Ullrich, J., \& Van Dick, R. (2013). Within-person variation in affective commitment to teams: Where it comes from and why it matters. Human Resource Management, 23(2), 131-147. doi: 10.1016/j.hrmr.2012.07.006

*Binning, J., Bradshaw, A., \& LeBreton J. (2010, Abril). Understanding turnover propensity via job-specific and identity-based emotional beliefs. Anais da 25th Annual Conference of the Society for Industrial and Organizational Psychology. Atlanta: Society for Industrial and Organizational Psychology.

*Breckler, S. J., \& Wiggins, E. C. (1989). Affect versus evaluation in the structure of attitudes. Journal of Experimental Social Psychology, 25, 253-271.

*Brief, A. P. (2001). Organizational behavior and the study of affect: Keep your eyes on the organization. Organizational Behavior and Human Decision Processes, 86(1), 131-139. doi: 10.1006/obhd.2001.2975

Brief, A., \& Weiss, H. M. (2002). Organizational behavior: Affect in the workplace. Annual Review of Psychology, 53, 279-307. doi: 10.1146/annurev. psych.53.100901.135156

Cammann, C., Fichman, M., Jenkins, G. D., \& Klesh, J. (1983). Michigan organizational assessment questionnaire. In S. E. Seashore, E. E. Lawler, P. H. Mirvis \& C. Cammann (Eds.), Assessing organizational change: A guide to methods, measures, and practices (pp. 71-138). New York: Wiley-Interscience.

*Carlson, D., Kacmar, M., Zivnuska, S., Ferguson, M., \& Whitten, D. (2011). Workfamily enrichment and job performance: A constructive replication of affective events theory. Journal of Occupational Health Psychology, 16(3), 297-312. doi: $10.1037 / \mathrm{a} 0022880$
*Dalal, R., \& Credé, M. (2013). Job satisfaction and other job attitudes. In K. F. Geisinger (Ed.), APA handbook of testing and assessment in psychology, Vol. 1: Test theory and testing and assessment in industrial and organizational psychology (pp. 675-691). Washington: American Psychological Association. doi: $10.1037 / 14047-037$

*Donnelly, D. P., \& Quirin, J. J. (2006). An extension of Lee and Mitchell's unfolding model of voluntary turnover. Journal of Organizational Behavior, 27(1), 59-77. doi: 10.1002/job.367

Eagly, A. H., \& Chaiken, S. (1993). The psychology of attitudes. Fort Worth: Harcourt Brace Jovanovich College Publishers.

Fisher, C. D. (1997, Junho). Emotions at work: What do people feel and how should we measure it? Paper apresentado na Second Biennial Australian Industrial and Organisational Psychology Conference, Melbourne. Recuperado de http://epublications.bond.edu.au/business_pubs/1

*Fisher, C. D. (2000). Mood and emotions while working: Missing pieces of job satisfaction? Journal of Organizational Behavior, 21(2), 185-202. doi: 10.1002/ (SICI)1099-1379(200003)21:2<185::AID-JOB34>3.0.CO;2-M

Fisher, C. D. (2002). Real time affect at work: A neglected phenomenon in organisational behaviour. Australian Journal of Management, 27, 1-10.

*Fredrickson, B. L., Tugade, M. M., Waugh, C. E., \& Larkin, G. R. (2003). What good are positive emotions in crisis? A prospective study of resilience and emotions following the terrorist attacks on the United States on September 11th, 2001. Journal of Personality and Social Psychology, 84(2), 365-376. doi: 10.1037/0022-3514.84.2.365

Frijda. N. H. (1993). Moods, emotion episodes and emotions. In M. Lewis \& J. M Haviland (Eds.), Handbook of emotions (pp. 381-403). New York: Guillford Press.

Grandey, A. A., Tam, A. P., \& Brauburger, A. L. (2002). Affective states and traits in the workplace: Diary and survey data from young workers. Motivation and Emotion, 26(1), 31-55. doi: 10.1023/A:1015142124306

Gooty, J., Gavin, M. B., Ashkanasy, N. M., \& Thomas, J. S. (2014). The wisdom of letting go and performance: The moderating role of emotional intelligence and discrete emotions. Journal of Occupational and Organizational Psychology, 87, 392-413. doi: 10.1111/joop.12053

*Gross, S., Semmer, N. K., Meier, L. L., Kälin, W., Jacobshagen, N., \& Tschan, F. (2011). The effect of positive events at work on after-work fatigue: They matter most in face of adversity. Journal of Applied Psychology, 96(3), 654-664. doi: 10.1037/a0022992

*Holtom, B. C., Burton, J. P., \& Crossley, C. D. (2012). How negative affectivity moderates the relationship between shocks, embeddedness and worker behaviors. Journal of Vocational Behavior, 80, 434-443. doi: 10.1016/j. jvb.2011.12.006

*Humphrey, S. E., Nahrgang, J. D., \& Morgeson, F. P. (2007). Integrating motivational, social, and contextual work design features: A meta-analytic summary and theoretical extension of the work design literature. Journal of Applied Psychology, 92(5), 1332-1356. doi: 10.1037/0021-9010.92.5.1332

*Hu, X., \& Kaplan, S. (2014). Is "feeling good" good enough? Differentiating discrete positive emotions at work. Journal of Organizational Behavior, 36(1), 39-58. doi: 10.1002/job.1941

Ilies, R., \& Judge, T. A. (2002). Understanding the dynamic relationships among personality, mood and job satisfaction: A field experience sampling study. Organizational Behavior and Human Decision Processes, 89, 1119-1139. doi: 10.1016/S0749-5978(02)00018-3

*Isen, A. M., \& Baron, R. A. (1991). Positive affect as a factor in organizational behavior. En C. Hendrick (Ed.), Close relationships (pp. 1-53). Greenwich: JAI Press.

Iverson, R., \& Deery, S. (2001). Understanding the "personological" basis of employee withdrawal: The influence of affective disposition on employee tardiness, early departure, and absenteeism. Journal of Applied Psychology, 86(5), 856-866. doi: 10.1037/0021-9010.86.5.856

Kohn, M. L., \& Schooler, C. (1982). Job conditions and personality: A longitudinal assessment of their reciprocal effects. The American Journal of Sociology, 87(6), 1257-1286.

Kozlowski, S. W. J. (Ed.) (2012). The oxford handbook of organizational psychology (Vol. 2). New York: Oxford University Press.

Lazarus, R. S. (1991). Emotion and adaptation. New York: Oxford University Press. Lee, K., \& Allen, N. (2002). Organizational citizenship behavior and workplace deviance: The role of affect and cognitions. Journal of Applied Psychology, 87(1), 131-142. doi: 10.1037/0021-9010.87.1.131

LeBreton, J., Binning, J., Adorno, A., \& Melcher, K. (2004). Importance of personality and job-specific affect for predicting job attitudes and withdrawal behavior. Organizational Research Methods, 7, 300-325. doi: $10.1177 / 1094428104266015$ 
Levine, E., Xu, X., Yang, L., Ispas, D., Pitariu, H., Bian, R.,...Musat, S. (2011). Cross-national explorations of the impact of affect at work using the state-trait emotion measure: A coordinated series of studies in the United States, China, and Romania. Human Performance, 24(5), 405-442. doi: 10.1080/08959285.2011.614302.

*Lindebaum, D., \& Jordan, P. (2014). When it can be good to feel bad and bad to feel good: Exploring asymmetries in workplace emotional outcomes. Human Relations, 67(9), 1037-1050. doi: 10.1177/0018726714535824

Miralles, C., Navarro, J., \& Unger, D. (2015) Daily work events and state work engagement: The mediating role of affect. Revista Psicología Social, 30(2), 264-294. doi: 10.1080/21711976.2015.1016755.

*Morris, W. N. (1989). Springer series in social psychology. Mood: The frame of mind. New York: Springer-Verlag Publishing.

Navarro, J., Roe, R. A., \& Artiles, M. I. (2015). Taking time seriously: Changing practices and perspectives in Work/Organizational Psychology. Journal of Work and Organizational Psychology, 31(3), 135-145. doi: http://dx.doi. org/10.1016/j.rpto.2015.07.002

*Ouweneel, E., Le Blanc, P., Schaufeli, W., \& Van Wijhe, C. (2012). Good morning, good day: A diary study on positive emotions, hope, and work engagement. Human Relations, 65(9), 1129-1154. doi: 10.1177/0018726711429382.

Ortony, A., Clore, G. L., \& Collins, A. (1998). The cognitive structure of emotions. Cambridge: Cambridge University Press.

Paterson, J., \& Cary, J. (2002). Organizational justice, change anxiety, and acceptance of downsizing: Preliminary tests of an AET- based model. Motivation and Emotion, 26(1), 83-103. doi: 10.1023/A:1015146225215

Roe, R. A. (2008). Time in applied psychology. The study of what happens rather than what is. European Psychologist, 13(1), 37-52.

*Rothbard, N., \& Wilk, S. (2011). Waking up on the right or wrong side of the bed: Start-of-workday mood, work events, employee affect, and performance. Academy of Management Journal, 54(5), 959-980. doi: 10.5465/ amj.2007.0056.

*Schaufeli, W. B., Salanova, M., González-Romá, V., \& Bakker, A. B. (2002). The measurement of engagement and burnout: A confirmative analytic approach. Journal of Happiness Studies, 3(1), 71-92. doi: 10.1023/A:1015630930326

Sheldon, K. M., \& Lyubomirsky, S. (2006). How to increase and sustain positive emotion: The effect of expressing gratitude and visualizing best possible selves. Journal of Positive Psychology, 1(2), 73-82. doi: 10.1080/17439760500510676

*Schlett, C., \& Ziegler, R. (2013). Job emotions and job cognitions as determinants of job satisfaction: The moderating, role of individual differences in need for affect. Journal of Vocational Behavior, 84(1), 74-89. doi: 10.1016/j. jvb.2013.11.005

Shockley, K., Ispas, D., Rossi, M., \& Levine, E. (2012). A meta-analytic investigation of the relationship between state affect, discrete emotions, and job performance. Human Performance, 25(5), 377-411. doi: 10.1080/08959285.2012.721832

Smith, C. A., \& Pope, L. K. (1992). Appraisal and emotion: The interactional contributions of dispositional and situational factors. In M. S. Clark (Ed.), Review of personality and social psychology, Vol. 14. Emotion and social behavior (pp. 32-62). Thousand Oaks: Sage Publications.

*Totterdell, P., \& Niven K. (2014). Workplace moods and emotions: A review of research. Charleston: Createspace Independent Publishing, 31.

*Warr, P., \& Clapperton, G. (2010). The joy of work? Jobs, happiness, and you. New York: Routledge/Taylor \& Francis Group.

Watson, D., \& Clark, L. A. (1994). The PANAS-X: Manual for the positive and negative affect schedule - Expanded Form. lowa City: University of lowa.

Watson, D., Clark, L. A., \& Tellegen, A. (1988). Development and validation of brief measures of positive and negative affect: The PANAS scales. Journal of Personality and Social Psychology, 54(6), 1063-1070. doi: 10.1037/0022-3514.54.6.1063

*Weiss, H. M. (2002). Deconstructing job satisfaction: Separating evaluations, beliefs and affective experiences. Human Resource Management Review, 12(2), 173-194. doi: 10.1016/S1053-4822(02)00045-1

*Weiss, H. M. (2013). Working as human nature. In Ford, J. K. (Ed.), The nature of work: Advances in psychological theory, methods, and practice (pp. 35-47). Washington: American Psychological Association. doi: 10.1037/14259-003

Weiss, H. M., \& Beal D. J. (2005). Reflections on affective events theory. Research on Emotion in Organizations, 1, 1-21. doi: I0.1016/S1746-9791(05)01101-6

Weiss, H. M., \& Cropanzano, R. (1996). Affective events theory: A theoretical discussion of the structure, causes and consequences of affective experiences at work. In B. M. Staw \& L. L. Cummings (Eds.), Research in organizational behavior: An annual series of analytical essays and critical reviews (Vol. 18, pp. 1-74). Greenwich: Elsevier Science/JAI Press.

Weiss, H. M., \& Rupp, D. E. (2011). Experiencing work: An essay on a person-centric work psychology. Industrial and Organizational Psychology, 4(1), 83-97. doi: 10.1111/j.1754-9434.2010.01302.x

Wilson, T. D., \& Hodges, S. D. (1992). Attitudes as temporary constructions. In L. Martin \& A. Tesser (Eds.), The construction of social judgments (pp. 37-65). Hillsdale: Lawrence Erlbaum Associates.

*Zaho, H., Wayne, S., Glibkowski, B., \& Bravo, J. (2007). The impact of psychological contract breach on work- related outcomes: A meta-analysis. Personnel Psychology, 60(3), 647-680. doi: 10.1111/j.1744-6570.2007.00087.x

*Ziegler, R., Schlett, C., Casel, K., \& Diehl, M. (2012). The role of job satisfaction, job ambivalence, and emotions at work in predicting organizational citizenship behavior. Journal of Personnel Psychology, 11, 176-190. doi: 10.1027/1866-5888/a000071

Zohar, D., Tzischinski, O., \& Epstein, R. (2003). Effects of energy availability on immediate and delayed emotional reactions to work events. Journal of Applied Psychology, 88(6), 1082-1093. doi: 10.1037/0021-9010.88.6.1082

* Las referencias marcadas con un asterisco indican aquellas referencias que se han incluido en la revisión exhaustiva presentada en este artículo. 